\title{
Possibilities of Using Tracking Methods for Trains in the Czech Republic
}

\author{
Jiř́ Hanzl ${ }^{1}$, Ladislav Bartuška ${ }^{1, *}$, Josef Šedivý ${ }^{1}$, Tomáš Kůs ${ }^{1}$, Martin Kůs $^{1}$ and Jiř́ Novotný ${ }^{1}$ \\ ${ }^{1}$ Institute of Technology and Business in České Budějovice, Department of Transport and Logistics, Okružní 517/10, 37001 České \\ Budějovice, Czech Republic
}

\begin{abstract}
The aim of this paper is to familiarize readers with several telematic systems that may be observed on the Czech railway network. In order to function correctly, they use special technical devices for detecting positions of trains and their tracking. In the introductory part, a brief overview of individual ways of train detecting and tracking in the Czech Republic is given, which is then followed by a detailed description of the related telematic systems, including the principles of their functioning and graphic presentation of their practical use. The paper also contains demonstrative photo documentation and schematic illustrations of principles of functioning of the discussed systems.
\end{abstract}

\section{Introduction}

Devices for detecting positions of trains and their tracking are one of the basic sources of information for different systems used on railways. These may include, for instance, various information and navigation systems that inform passengers at stations or cargo carriers about the current positions of trains on railway tracks and their delays or automated train management systems that are capable (based on the data obtained from detectors about the positions of trains) of automating and optimizing their journey with regard to minimum traction power consumption, adhering to the planned journey time without delays and indeed safe railway vehicle stopping. Within the Czech railway network, one may come across four basic ways of train detecting and tracking - axle counters, track circuits, magnetic information points and eurobalises. In the next part, the previously mentioned ways are briefly described and then the related telematic systems used on the Czech railway network are introduced.

\section{Devices for detecting positions of trains and their tracking}

Certain ways of detecting positions of trains have been applied particularly in the context of increasing requirements for railway safety and increasing the capacity of the railway infrastructure. At present, automatic or semiautomatic devices are largely used for train tracking, and depending on their technical design and function, they are divided into the point devices and the continuous devices. In the Czech Republic, it is possible to observe four basic ways of detecting positions of trains - axle counters, track circuits, eurobalises and magnetic information points.
Axle counters are devices for detecting railway vehicles on particular sections of railway tracks using point units, such as wheel detectors. They are therefore point-tracking devices made up of two parts, i.e. an outer part and an inner part. The outer part consists of the wheel detectors, whilst the inner part comprises a device which (on the basis of information from the outer part) is able to recognize whether railway vehicles are or are not located on the given sections of railway tracks. On the other hand, track circuits are used to detect railway vehicles on particular sections of railway tracks by means of the electric circuit and changes in ratios therein [1]. Thus, these are continuous tracking devices and each track circuit consists of a track line and connected equipment, with the track line encompassing a railway upper, where rails form wires and sleepers and gravel serve as insulation. Track circuits can be divided into two basic types, i.e. series and parallel wiring circuits. Both of these types work on the principle of changing the magnitude of the electric current that takes place in the circuit. The track circuit components are constituted by an electric power source, an adjustable resistor and an intrinsically safe relay (with an armature in the relay dropping off when the power is interrupted). As for the so-called eurobalises, positions of trains are detected through point units intended for an inductive transmission of information between railway vehicles and railway tracks within the ETCS (European Train Controlling System, see Subchapter 3.2). Some eurobalises contain information about their exact position and serve as reference points for passing railway vehicles. On their passing over the balises, information about the position is then transferred to the vehicles via a transmission module placed on them. Lastly, magnetic information points are used to detect positions of railway vehicles within the CRV and AVV systems. The

Corresponding author: bartuska.vste@,seznam.cz 
information about positions obtained from such points is intended solely for the purposes of these systems. Hence, there is only way of how to use them - magnetic information points have data about their exact positions encoded through combining differently placed magnets and this information is transferred to railway vehicles on their passing over the magnetic information points via sensors placed on the vehicles [2].

\section{Possibilities of using tracking methods for trains}

As stated in the previous chapter, technical devices for train tracking represent an important source of information for all modern security equipment that (based on train positioning data) ensure safe and continuous operating on the rail network. These are not only station, rail line and crossing security devices, but mainly devices designed to check compliance with driving parameters, and (in the event of their nonobservance) to directly intervene in train control. The following subchapters (3.1 and 3.2) are focused on two train security systems (LS and ETCS) which are linked to Czech trains.

\subsection{LS system}

The LS system is seen as a continuous train security system that was developed in former Czechoslovakia back in the 1960's and has since been modified several times. Currently, the LS 90 version is used [3]. However, one may not rely too much on further development of the system, particularly due to gradual deployment of the ETCS (see Subchapter 3.2), which is to sequentially replace different train security systems in other countries as well. The main function of the LS system is to transfer a signal via track circuits to a railway vehicle's cabin (cab), where the signal is displayed on a signal repeater (Fig. 1.).

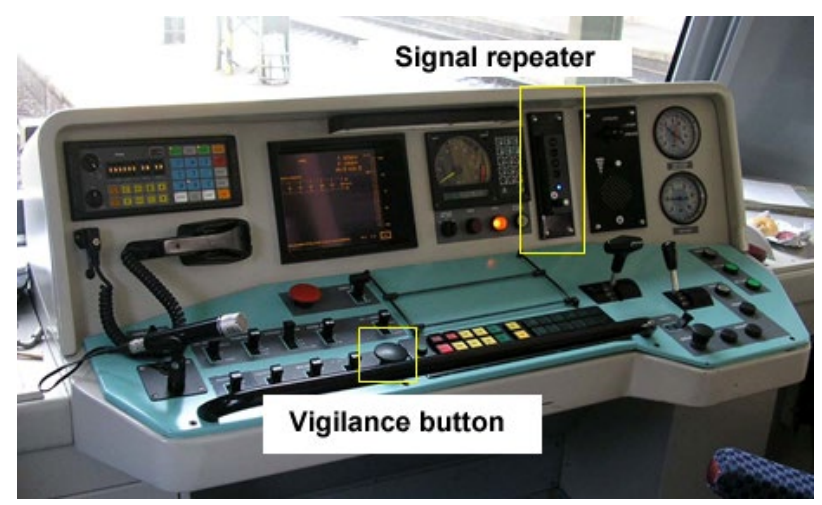

Fig. 1. Control panel with the LS system. [4]

Thus, train drivers are able to see the signal on the following signal device at the beginning of a particular section. The system also works with the so-called vigilance device (button), and while driving on railway tracks not having the LS, train drivers must press the button every 20 seconds. On the contrary, its pressing on the LS railway tracks is only required when the next signal device indicates a red "Stop" light or a yellow "Deceleration" sign (Fig. 2.).

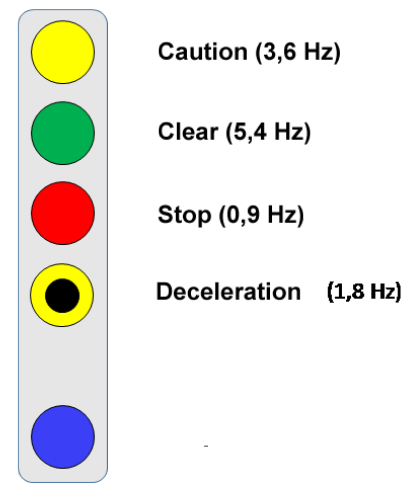

Fig. 2. Signal device - frequencies for individual signals.

The LS system consists of a trackside part and an onboard part. The former comprises independent track circuits with an encoder that transmits a code with information about the current signal via track circuits. On the other hand, the latter allows to transfer information from a signal on a given railway track to a driving vehicle and to check its driver's vigilance, and is made up of scanning coils, a signal repeater and a vigilance device (button). The coils are located at the front of the vehicle and are used to capture the code transmitted by the encoder via track circuits. The signal repeater then visually communicates information about the upcoming signal to the driver [5].

The signal transmitting encoder is usually located at a respective signal device from which it receives data and current signals. The data are then converted by the encoder into a code of a certain frequency corresponding to a particular signal. The code is subsequently transmitted by track circuits, and upon a railway vehicle entering a given railway track section, the circuit is connected via conductive axles by which there are attached coils able to read the transmitted code [6]. Afterwards, this code is processed and the information about the current signal is transferred to the signal repeater. The LS system functioning is schematically shown in Fig. 3.

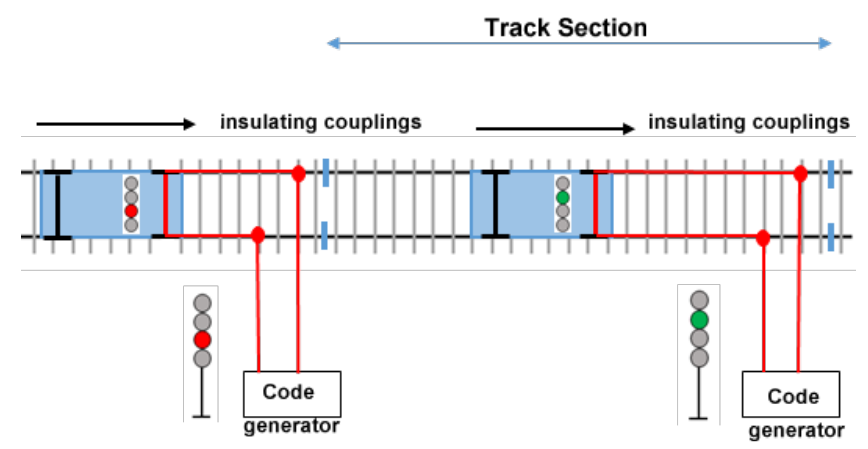

Fig. 3. Principle of the LS system - schematic illustration.

\subsection{ETCS}

The European Train Control System (ETCS) is part of the ERTMS project. This project was established to 
develop a unified security system that would replace various security systems in individual European countries, thereby achieving interoperability on the European rail network and increasing its competitiveness. The ERTMS has two basic subsystems, i.e. the aforementioned ETCS and the international GSM-R (Global System for Mobile CommunicationsRailway) for wireless voice and data communication between railway vehicles and dispatch centers [7].

To put it simply, the main principle of fully-fledged ETCS functioning resides in trains knowing their exact position on railway tracks, which is continually reported to dispatch centers. They are then aware of positions of all trains within the network and issue the so-called driving authorization for each train, which is the permission for the trains to be driven to precisely defined destinations (complemented by possible restrictions on driving in particular sections). Based on the permission, individual trains control their speed or stop to avoid collision with other trains. Thus, the main functions of the ETCS include controls of driving authorizations, controls of perceiving and respecting the restrictions by drivers and full controls of drivers and their work. The ETCS consists of two parts - a trackside part and an on-board part.

The trackside part involves eurobalises transferring information between railway tracks and railway vehicles, LEUs (Lineside Electronic Units), RBC (Radio Block Center), euroloops and additional radio circuit. The onboard part encompasses EVC (European Vital Computer), JRU (Juridical Recording Unit), the DMI imaging unit (see Fig. 4), BTM (Balise Transmission Module) and a set of equipment for accurate detecting of the speed of vehicles, including their odometry.

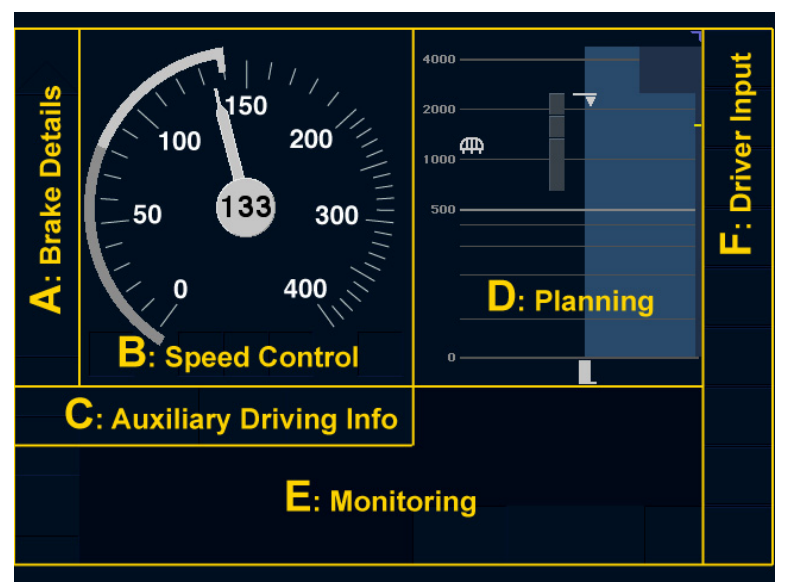

Fig. 4. DMI imaging unit. [8]

At present, there are three ETCS levels differing from each other by the extent of the ETCS involvement as train security equipment. Dividing the system into individual levels facilitates its gradual implementation and replacement of various security systems in other countries. At lower levels, the ETCS rather complements the already existing devices, yet it is gradually taking over their functions with its increasing level.

\subsubsection{Level 1 (L1)}

It is the lowest level of the ETCS involvement complementing the already used security devices. Controlling the availability of rail sections is still ensured by standard ways, such as the above mentioned track circuits or axle counters. However, railway tracks are also fitted with fixed and switchable balises. The fixed balises serve as reference points passing information about positions to railway vehicles as well as a source of information about invariable railway track characteristics. The switchable balises are connected through cables with the LEUs that are interconnected with security devices, thereby obtaining information about the railway track availability and the current signal of signal devices that rail vehicles are approaching [9].

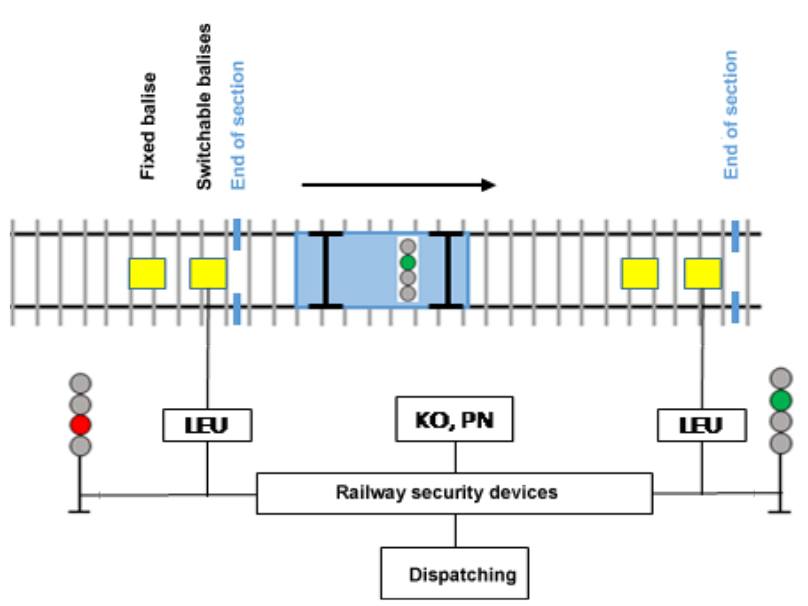

Fig. 5. Principle of the ETCS L1 - schematic illustration.

Subsequently, all the data are transferred via the switchable balises to railway vehicles, whereby their EVCs calculate the maximum speed and braking points. The signal data are transferred to railway vehicles on their passing over the balises and are not changed since then. Thus, in case there are any changes in the signal after the vehicles have passed over the balises, this will be detected by their drivers from the respective signal device. Solving this issue may reside in using the aforementioned euroloops (instead of the balises) between the "Caution" signal and the main signal device as these (unlike the balises) pass information to vehicles continuously [9].

Trackside functions of L1:

- authorization to drive (driving authorization) over switchable balises according to block sections based on data from security devices (Fig. 6),

- transfer of driving authorization and railway track description to railway vehicles. 


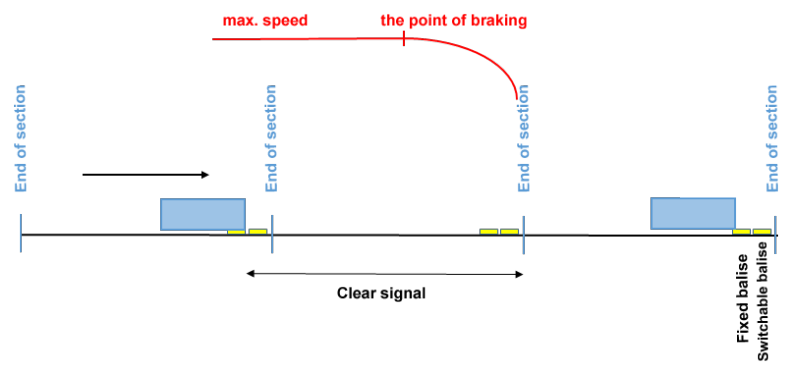

Fig. 6. Driving authorization in ETCS L1 - schematic illustration.

On-board functions of L1:

- receiving driving authorization and railway track description from balises,

- calculation of dynamic speed profile,

- comparing the current speed of railway vehicles with the permitted speed, possible application of brakes,

- on-board signaling for train drivers [10].

\subsubsection{Level 2 (L2)}

The L2 uses only the fixed eurobalises serving as reference points. Using the switchable balises is not required since all the necessary information (driving authorization, railway track description) is continuously transferred to railway vehicles via the GSM-R from RBC which continuously observes positions of railway vehicles and is in communication with a security device monitoring the availability of railway track sections. Based on these data, RBC (through the GSM-R) issues driving authorization to railway vehicles. Owing to the continuous communication between $\mathrm{RBC}$ and the railway vehicles, it is no longer necessary to use signal devices on railway tracks.

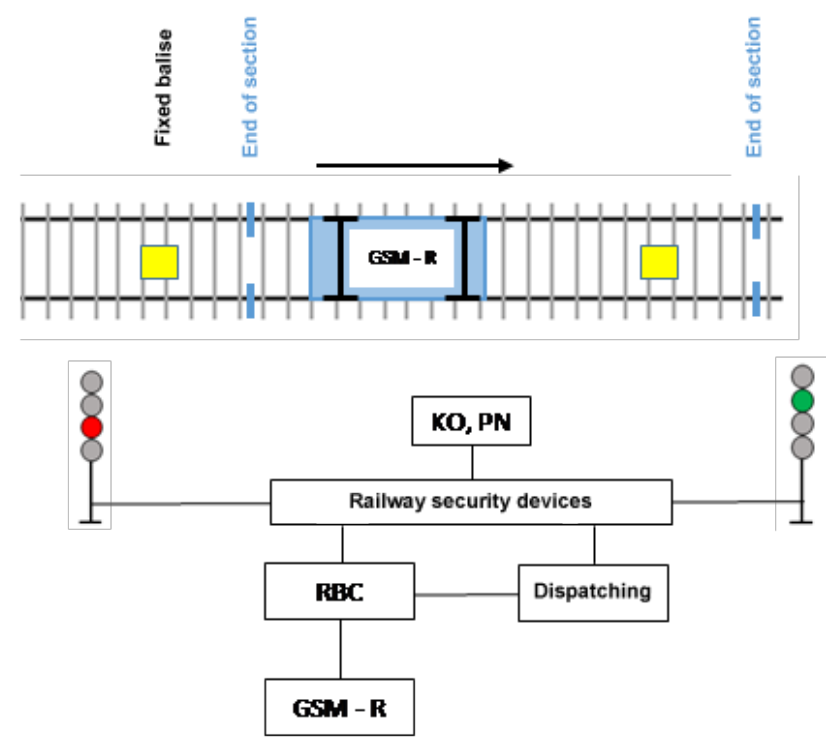

Fig. 7. Principle of ETCS L2 functioning - schematic illustration.

Trackside functions of L2:

- registration of all trains equipped with the ETCS in $\mathrm{RBC}$
- tracking of all trains equipped with the ETCS in $\mathrm{RBC}$,

- authorization to drive issued by $\mathrm{RBC}$ according to block sections based on data from security devices (Fig. 8),

- continuous transfer of driving authorization and railway track description to railway vehicles via the GSM-R.

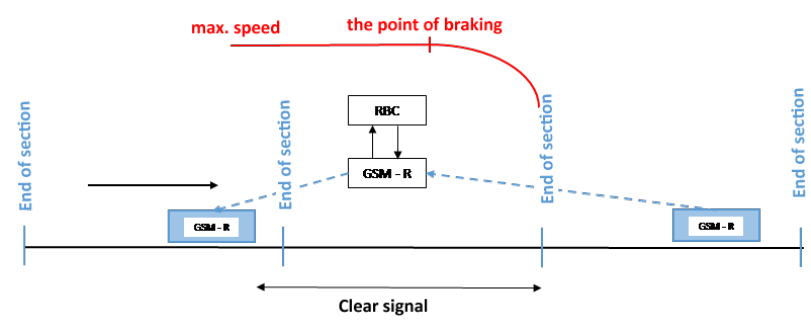

Fig. 8. Driving authorization in ETCS L2 - schematic illustration.

On-board functions of L2:

- transmitting positions to RBC via the GSM-R,

- calculation of dynamic speed profile,

- comparing the current speed of railway vehicles with the permitted speed, possible application of brakes,

- on-board signaling for train drivers [10].

\subsubsection{Level 3 (L3)}

It is the highest level of the ETCS where train security is provided exclusively by the ETCS as such without additional security devices and without fixed units that are replaced by virtual units. Railway tracks are equipped with fixed balises, again serving as reference points for the calibration of data about positions of railway vehicles. The $\mathrm{RBC}$ system continuously monitors the movement of all railway vehicles on railway tracks and issues driving authorization and other necessary information [11-13].

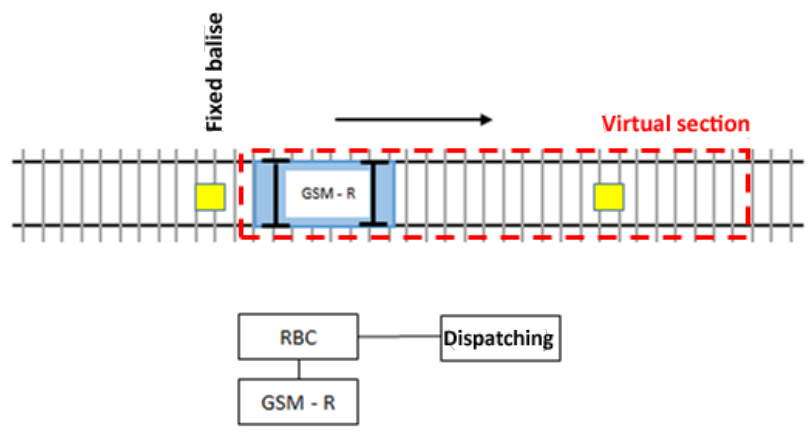

Fig. 9. Principle of ETCS L3 functioning - schematic illustration

Trackside functions of L3:

- registration of all trains equipped with the ETCS in $\mathrm{RBC}$,

- tracking of all trains equipped with the ETCS in $\mathrm{RBC}$,

- determining the end of a journey and its cancelling in accordance with information from railway vehicles, 
- driving authorization by $\mathrm{RBC}$ up to the last reported position of the previous train based on tracking positions of all railway vehicles on railway tracks (Fig. 10),

- continuous transfer of driving authorization and railway track description to railway vehicles via the GSM-R.

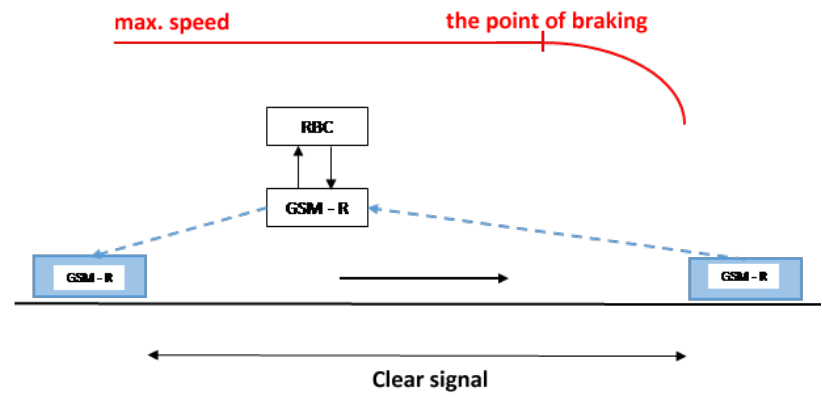

Fig. 10. Driving authorization in ETCS L3 - schematic illustration.

On-board functions of L3:

- transmitting positions to RBC via the GSM-R,

- trains monitor their integrity and transmit it to RBC,

- calculation of dynamic speed profile,

- comparing the current speed of railway vehicles with the permitted speed, possible application of brakes,

- on-board signaling for train drivers [14].

\section{Conclusion}

The aim of the paper was to acquaint readers with train tracking devices and describe selected telematics systems used on the Czech railway network. In the introductory part, particular ways of detecting positions of trains on railway tracks were briefly described, whilst the following chapters were related to train security devices - the LS system and the European Train Control System (ETCS). Both systems differ from each other by security levels and ways of functioning. The main function of the LS system is to transfer signals to cabins of railway vehicles and to check their drivers' vigilance by means of a special device (button). In order to transfer information about the status of signals to railway vehicles, the system uses track circuits. The main function of the ETCS is to issue driving authorization and to check the security of the trains being operated based on the input data from railway tracks, the system is able to control the speed of trains or possibly even stop them. The system comes in three levels depending on the extent of its involvement in train security, yet only the Level 1 and the Level 2 systems are used in the Czech Republic [13]. The Level 3 system works completely independently without fixed units that are replaced by virtual units the ETCS itself creates. Due to technically more demanding solutions, however, this is mainly reflected in high acquisition and operating costs of this system.

The paper originated within the project "Research for applying practical skills on software specific for students of logistics disciplines" as a Specific university research on the Institute of Technology and Business in Ceske Budejovice.

\section{References}

1. J. Gašparík, B. Abramović, V. Zitrický. Tehnicki Vjesnik,. 25, 4. 1190-1195, (2018)

2. V. Chudáček a kol., Detection of trains in railway security technology (in Czech), II. Edition (2015). Available

on: http://zabzar.cz/sites/default/files/KO_n.pdf (2005)

3. L. Ferfecki, ETCS Train Alarm System: bachelor thesis. Pardubice: University of Pardubice. (2010)

4. Atlas of locomotives (in Czech). 471 (2018). Available on: http://www.atlaslokomotiv.net/loko471.html

5. T. Dlask, Design of a cooperative system for communication at railway crossings: Diploma thesis. Prague: Czech Technical University in Prague. (2005)

6. V. Luptak, J. Gasparik, J., M. Chovancova, MATEC Web of Conferences. 134. Article number 0003318th. International Scientific Conference, LOGI 2017. (2017)

7. P. Pinkas. Journal Automa 1 (2014). Available on: http://automa.cz/res/pdf/52955.pdf

8. J. Klimkova, J. Camaj, J. Gasparík. Transport means 2016: proceedings of the 20th international scientific conference. 891-896. (2016)

9. TORC2U [online]. TRAIN ELECTRONICS. Available on: http://torc2u.com.au/electronics/

10. T. Konopac, ERTMS/ETCS: semester work. Prague: Czech Technical University in Prague. (2010)

11. J. Sedivy, Possibilities of Using Tracking Methods for Trains in the Czech Republic: semester work. České Budějovice: Vysoká škola technická a ekonomická v Českých Budějovicích. (2017)

12. V. Luptak, O. Stopka, K. Jerabek, MATEC Web of Conferences, 134, Article number 00034, 18th International Scientific Conference, LOGI 2017; (2017)

13. J. Masek, P. Kolarovszki, J. Camaj, Procedia Engineering, 134. 231-236 (2016)

14. A. Dolinayova, L. Cerna, V. Zitricky, The role of railway transport in east-west traffic flow conditions of the Slovak Republic. 121-169 (2018) 